\title{
Semiconductor Optical Amplifiers
}

\author{
M. Haridim, B.I. Lembrikov, Y. Ben-Ezra \\ Holon Institute of Technology (HIT),52 Golomb Str., Holon 58102
}

Israel

\section{Introduction}

The theoretical and experimental studies of semiconductor optical amplifiers (SOAs) started immediately after the invention of semiconductor lasers as early as in 1962, but practical applications of SOAs began in the 1980s when SOAs have emerged as an important component in many optical fiber communication systems Agrawal (2002). The applications of SOAs in optical communications, switching and signal processing based on their high performance manifested in laboratory prototypes were predicted as early as 1989 Eisenstein (1989). SOA is characterized by extremely strong non-linearity, low power, high operation rate, and small size as compared to erbium doped fiber amplifiers (EDFAs) and Raman optical amplifiers. The cross gain modulation (XGM), cross phase modulation (XPM), four-wave mixing (FWM) phenomena are strongly manifested in SOA Agrawal (2002).

The two main application areas of SOA are the linear in-line amplification in gigabit passive optical networks (GPON), and fast nonlinear all-optical signal processing Freude (2010). In particular, SOAs are among the most promising candidates for all-optical processing devices due to their high-speed capability, low switching energy, compactness, and optical integration compatibility Dong (2008). Hence, besides its use as an in-line optical amplifier, SOA provides a wide range of functional applications including wavelength conversion (WC), regeneration, photonic switching and various all-optical signal processing components.

High-speed WC, all-optical logic operations, and signal regeneration are important operations in all-optical signal processing where SOAs are widely used Agrawal (2002), Ramamurthy (2001), Dong (2008), Sun (2005). WC is essential for optical wavelength division multiplexing (WDM) network operation Ramamurthy (2001). There exist several all-optical techniques for wavelength conversion based on SOAs using XGM and XPM effects between a pulsed signal and a continuous wave $(\mathrm{CW})$ beam at a desired wavelength of the converted signal Agrawal (2002).

All-optical logic gates operation is based on nonlinearities of optical fibers and SOAs. However, optical fibers suffer from disadvantages such as weak nonlinearity, long interaction length, and/or high control energy required in order to achieve a reasonable switching efficiency Sun (2005). SOA, on the other hand, has high nonlinearity, small dimensions, low energy consumption, high operation speed, and can be easily integrated into photonic and electronic systems Sun (2005), Hamié (2002), Kanellos (2007), Dong (2008).

The major challenges of improving optical transmission systems stem mainly from signal-to-noise ratio (SNR) degradation and chromatic dispersion. For this reason, the optical signal reamplification, reshaping, and retiming, i.e. the so-called $3 \mathrm{R}$ regeneration, are necessary in order to avoid accumulation of noise, crosstalk and nonlinear distortions 
and to provide a good signal quality for transmission over any path in all-optical networks Sartorius (2001), Zhu (2007), Leem (2006), Kanellos (2007). Optical regeneration technology is characterized by lower power, much more compact size, and can provide transparency in the needed region of spectrum Zhu (2007). All-optical 3R regeneration is also less complex, provides better performance and uses fewer optoelectronic/electronic components than electrical regeneration Leem (2006), Kanellos (2007). SOA chips and packaged SOAs are used as tunable lasers with ultra-wide tuning range and $\mathrm{kHz}$ scan rate for sensing and medical applications Spiekman (2009). The wavelength agility and low power requirements of SOA stimulate their use in telecommunications as a range extender in Fiber to the Home networks and in transmission system optical channels outside the EDFA band Spiekman (2009).

Tunable optical delay lines and continuously controllable phase shifters for microwave signal processing are based on the slow and fast light propagation Dúill (2009). One of the most efficient mechanisms for the slow and fast light propagation is the coherent population oscillations (CPO) in SOAs due to its strongly manifested XPM, XGM and FWM Chang-Hasnain (2006), Dúill (2009).

Recent advances in quantum well (QW) SOAs, quantum dash (Q-dash) SOAs and especially quantum dot (QD) SOAs make them promising candidates for use in evolving optical fiber communication systems since a bulk SOA performance may be substantially improved in terms of threshold current density, saturation power, gain bandwidth, and the temperature dependence of quantum devices Bimberg (1999), Sugawara (2004), Ustinov (2003). The possibility of integrating into Si photonic technology makes QD SOA an especially important candidate for integrated electronic and photonic circuits on the same silicon platform.

The chapter is constructed as follows. The operation principle and the phenomenological theory of bulk SOA is presented in Section 2. Structure, dynamics and peculiarities of QW and QD SOAs are discussed in Section 3. Novel applications of SOAs such as photonic pulse generation, all-optical signal processing, all-optical logics, slow and fast light generation are reviewed in Section 4. Conclusions are presented in Section 5.

\section{Theory of bulk SOA}

\subsection{Structure and operation principle of bulk SOA}

In this section, we consider briefly the operation principle and the structure of a bulk SOA Eisenstein (1989), Agrawal (2002), Chang (2005). SOAs amplify incident light by the stimulated emission process using the same mechanism as laser diodes Agrawal (2002). In fact, SOA can be characterized as a semiconductor laser without feedback Agrawal (2002). Optical gain can be realized when the electrically or optically pumped SOA achieves population inversion Agrawal (2002). The optical gain depends on the incident signal frequency $\omega$ and the local beam intensity at any point inside the amplifier Agrawal (2002).

Bulk SOA consists of a laser diode with low facet reflectivities. Typically, the device contains several layers Eisenstein (1989), Agrawal (2002). Carriers from an external bias circuit are injected into the active region of SOA where they are confined by layers of materials with higher energy band gap. An optical signal impinging on the active region will induce simulated emission and will be amplified under the condition of population inversion, i.e. when the bias current is sufficiently large Eisenstein (1989). The active region serves as the core of a slab optical waveguide since its refractive index $n$ is larger than the refractive index of the cladding. In a semiconductor laser, the end facets are cleaved perpendicular to the waveguide forming mirrors to provide the feedback necessary for laser oscillations. The SOA structure is shown in Fig.1. 


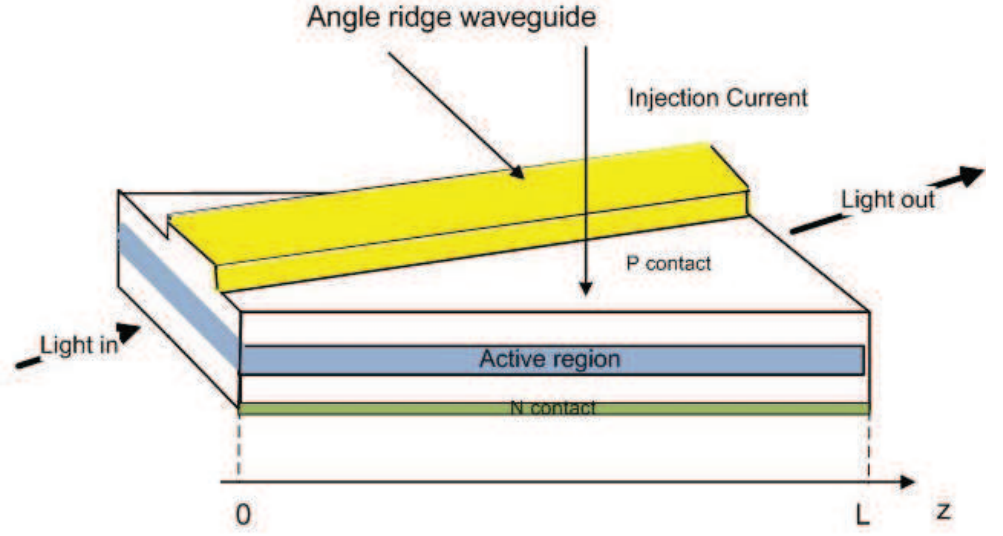

Fig. 1. Structure of SOA

SOA can operate in two different regimes. In the first case called the travelling-Wave (TW) regime, the oscillations are prevented in order to create a single pass gain, Eisenstein (1989), Agrawal (2002). The active medium is described as a homogeneously broadened two-level system. In such a case, the gain coefficient $g(\omega)$ has the form Agrawal (2002)

$$
g(\omega)=\frac{g_{0}}{1+\left(\omega-\omega_{0}\right)^{2} T_{2}^{2}+P / P_{s}}
$$

where $g_{0}$ is the peak value of the gain, $\omega_{0}$ is the transition frequency, $P$ is the optical power of the amplified signal, $P_{S}$ is the saturation power, and $T_{2} \leq 1 p s$ is the dipole relaxation time. Under the condition of $P / P_{S} \ll 1$ corresponding to the unsaturated regime, equation (1) takes the form

$$
g(\omega)=\frac{g_{0}}{1+\left(\omega-\omega_{0}\right)^{2} T_{2}^{2}}
$$

It is seen from equations (1) and (2) that the frequency dependence of the gain is characterized by a Lorentzian profile with the resonance frequency $\omega_{0}$. The gain bandwidth $\Delta \omega_{g}$ defined as the full width at half maximum (FWHM) is given by Agrawal (2002).

$$
\Delta \omega_{g}=\frac{2}{T_{2}}
$$

The local optical power $P(z)$ in a gain medium can be defined as Agrawal (2002).

$$
P(z)=P(0) \exp (g z)
$$

where $P(0)=P_{\text {in }}$ is the input optical power. We can define an amplification factor $G(\omega)$ for a SOA of length $L$ as the ratio between the output optical power $P_{\text {out }}=P(L)$ and $P_{\text {in }}$ Agrawal (2002).

$$
G(\omega)=\frac{P_{\text {out }}}{P_{\text {in }}}=\frac{P(L)}{P(0)}=\exp (g L)
$$

The gain saturation regime is defined by the gain dependence on the optical power according to equation (1). It can be shown that for the large-signal amplification factor the following 
relationship prevails Agrawal (2002).

$$
G=G_{0} \exp \left[-\frac{(G-1) P_{\text {out }}}{G P_{S}}\right]
$$

where $G_{0}$ is the unsaturated amplification factor. The main noise in SOA is due to the spontaneous emission. The SNR degradation is characterized by the amplifier noise figure (NF) $F_{n}$ defined by Agrawal (2002).

$$
F_{n}=\frac{(S N R)_{\text {in }}}{(S N R)_{\text {out }}}
$$

NF can reach $6-8 d B$. However for optical communication applications it should be as low as possible Agrawal (2002).

In the second regime, called Fabry-Perot (FP) SOA, a semiconductor laser biased slightly below threshold is used as SOA in a FP cavity where the FP cavity is characterized by multiple reflections at the facets with reflectivities $R_{1,2}$ Agrawal (2002). The amplification factor $G_{F P}(\omega)$ for such a FP SOA is given by Agrawal (2002)

$$
G_{F P}(v)=\frac{\left(1-R_{1}\right)\left(1-R_{2}\right) G}{\left(1-G \sqrt{R_{1} R_{2}}\right)^{2}+4 G \sqrt{R_{1} R_{2}} \sin ^{2}\left[\pi\left(v-v_{m}\right) / \Delta v_{L}\right]}
$$

where $v=\omega / 2 \pi, v_{m}=m c / 2 n L$ are the cavity resonance frequencies, $c$ is the free space light velocity, $\Delta v_{L}=c / 2 n L$ is the longitudinal mode spacing, and $m=1,2,3, \ldots$ is the mode number. The FP SOA bandwidth $\Delta v_{A}$ at the $3 d B$ level of $G_{F P}(v)$ is given by Agrawal (2002)

$$
\Delta v_{A}=\frac{2 \Delta v_{L}}{\pi} \arcsin \left[\frac{1-G \sqrt{R_{1} R_{2}}}{\left(4 G \sqrt{R_{1} R_{2}}\right)^{1 / 2}}\right]
$$

\subsection{Rate equations and optical field propagation equations}

The efficiency of SOA applications in all-optical integrated circuits for optical signal processing and functional devices is mainly determined by their high gain coefficient and a relatively low saturation power Agrawal (1989), Agrawal (2002), Premaratne (2008). Device engineering and performance optimization require simple and quantitatively accurate models for SOAs providing an adequate description of SOAs peculiarities Premaratne (2008).

The theory of pulse propagation in amplifiers based on a two-level system model is also valid for SOAs Agrawal (1989). The SOA's active region is modeled as an ensemble of noninteracting two-level systems with transition energies extending over the range of the conduction and valence bands Agrawal (1989). If the pulse width $T_{p} \gtrsim 1 p s$ is much larger than the intraband relaxation time $\tau_{\text {in }} \sim 0.1 p s$ and, on the other hand, $T_{p} \ll \tau_{e} \sim 10^{-10_{S}}$ where $\tau_{e}$ is the carrier lifetime, the rate-equation approximation can be applied where the amplification process within the SOA is described by the following rate equations for the carrier density $N(z, t)$ and the optical signal intensity $I(z, t)$ Agrawal (1989), Agrawal (2002), Premaratne (2008).

$$
\begin{gathered}
\frac{\partial N(z, t)}{\partial t}=\rho(z)-\frac{N(z, t)}{\tau_{e}}-g(z, t) \frac{\lambda I(z, t)}{h c} \\
\frac{\partial I(z, t)}{\partial z}+\frac{1}{v_{g}} \frac{\partial I(z, t)}{\partial t}=g(z, t) I(z, t)-\alpha I(z, t)
\end{gathered}
$$


where $t$ is the time, $z$ is the distance along the light propagation direction in the SOA active region, $\rho(z)$ is the current-injection density, $\alpha$ is the loss coefficient, $\lambda$ is the operating wavelength, and $h$ is the Planck's constant. The input pulse intensity profile $I_{0}(t)$ is given by Premaratne (2008)

$$
E_{g}=A_{m} \int_{-\infty}^{\infty} I_{0}(t) d t
$$

where $A_{m}$ is the effective mode area of the SOA active region, and $E_{g}$ is the pulse energy. The gain coefficient $g(z, t)$ is defined as Premaratne (2008).

$$
g(z, t)=\Gamma a\left[N(z, t)-N_{0}\right]
$$

where $\Gamma$ is the mode confinement factor, $a$ is the differential gain coefficient, and $N_{0}$ is the carrier density corresponding to the transparency state. By using the coordinate transformations $z, \tau=t-z / v_{g}$ and introducing the new variable

$$
h\left(z, \tau_{n}\right)=\int_{0}^{z} g(z, \tau) d z
$$

where $z \subset[0, L], \tau_{n}=\left(\tau v_{g}\right) / L$ is normalized time, and $L$ is the length of SOA, equations (10) and (11) can be reduced to the following integro-differential equation describing the gain recovery dynamics for a short optical pulse Premaratne (2008)

$$
\begin{aligned}
\frac{\partial h\left(z, \tau_{n}\right)}{\partial \tau_{n}}= & \epsilon\left[h_{\rho}-h\left(z, \tau_{n}\right)\right]-\beta\left(\tau_{n}\right)\left\{\exp \left[h\left(z, \tau_{n}\right)-\alpha z\right]-1\right\} \\
& -\beta\left(\tau_{n}\right)\left[\alpha \int_{0}^{z} \exp \left[h\left(z, \tau_{n}\right)-\alpha z\right] d z\right]
\end{aligned}
$$

where $\epsilon=L /\left(v_{g} \tau_{e}\right), \beta\left(\tau_{n}\right)=\Gamma a \lambda I_{0}(\tau) L /\left(v_{g} h c\right)$, and

$$
h_{\rho}(z)=\int_{0}^{z}\left[\tau_{e} \Gamma a \rho(z)-\Gamma a N_{0}\right] d z
$$

Equation (14) has been solved analytically and numerically for different situations using a multiple-scales technique Premaratne (2008). In particular, the signal gain $G(z, T, U)$ is given by Premaratne (2008).

$$
\begin{aligned}
& G(z, T, U) \exp (-\alpha z)=\exp \{[h(z)-\alpha z][1-\exp (-U)]\} \\
& \times\left\{1-\frac{1-\exp \left(-h_{I}(z)+\alpha z\right)}{E_{\gamma}(T)}\right\}^{-\exp (-U)}
\end{aligned}
$$

where $T=\tau_{n}$ is the pulse time scale, $U=\epsilon \tau_{n}$ is a slow time scale corresponding to the carrier recovery, and $h_{I}(z)$ is the initial profile of $h(z, T, U, \epsilon)$,

$$
E_{\gamma}(T)=\exp \left(\int_{0}^{T} \frac{\beta(T) \widetilde{g}(z, T, U)}{[\widetilde{g}(z, T, U)-\alpha]} d T\right)
$$




$$
\begin{aligned}
& \widetilde{g}(z, T, U)=\frac{h_{\rho}(z)}{z}[1-\exp (-U)]-\frac{\exp (-U)}{z} \\
& \times \ln \left[1-\frac{\left(1-\exp \left(-h_{I}(z)\right)\right)}{E_{\beta}(T)}\right]
\end{aligned}
$$

and

$$
E_{\beta}(T)=\exp \left(\int_{0}^{T} \beta(T) d T\right)
$$

The electric field $E(z, t)$ of the pulse is given by Premaratne (2008)

$$
E(z, t)=\sqrt{I(z, t)} \exp [i(\phi(z, t)-\omega t)] ; \phi(z, t)=\frac{\alpha_{L}}{2} h(z, t)
$$

where $\phi(z, t)$ is the phase of the pulse inside SOA and $\alpha_{L}$ is the linewidth enhancement factor (LEF).

Comparison of the analytical results of the theory developed above with the numerical simulation results for Gaussian picosecond pulses propagating through a SOA of length $L$ $\approx 400 \mu \mathrm{m}$, active region width and thickness of $2.5 \mu \mathrm{m}$ and $0.2 \mu \mathrm{m}$, respectively, $\Gamma=0.3$, carrier injection rate $1.177 \times 10^{34} \mathrm{~s}^{-1} \mathrm{~m}^{-3}, n_{g}=3.7, \alpha=3000 \mathrm{~m}^{-1}, \tau_{e}=3 \times 10^{-10} \mathrm{~s}, a=2.5 \times 10^{-20} \mathrm{~m}^{2}$, $N_{0}=1.5 \times 10^{24} \mathrm{~m}^{-3}, \alpha_{L}=5$, and $\lambda=1552.52 \mathrm{~nm}$ shows a good accord Premaratne (2008).

\section{Advanced structures}

\subsection{QW SOA}

The structure of QW SOA devices is very similar to that of bulk SOA, except that the active layer thickness of the former is reduced to the order of $10 \mathrm{~nm}$ where quantum effects play an essential role. A thin layer sandwiched between two layers of a wide band gap material form a finite potential well. As a consequence of carriers confinement in the $z$ direction in the thin layer, the conduction and valence bands break up into a series of well-defined sub-bands with a step-like density of states (DOS) function $\rho_{Q W}(E)$ which is energy independent. DOS function for electrons has the form Zhao (1999)

$$
\rho_{Q W}(E)=\sum_{n_{e}} \frac{m_{e}}{\pi \hbar^{2}} H\left[E_{e}-E_{e z}\left(n_{e}\right)\right]
$$

where $m_{e}$ is the electron effective mass, $\hbar=h /(2 \pi), E_{e}$ is the total electron energy given by

$$
E_{e}=E_{e z}\left(n_{e}\right)+\frac{(\hbar k)^{2}}{2 m_{e}}
$$

$E_{e z}\left(n_{e}\right)$ are the quantized energy levels, $n_{e}=1,2, \ldots$ is the level number, $k=\sqrt{k_{x}^{2}+k_{y}^{2}}$ is the electron wave vector corresponding to the motion in the $(x, y)$ directions, and $H[x]$ is the Heaviside function given by

$$
H[x]=\left\{\begin{array}{l}
1, x \geq 0 \\
0, x<0
\end{array}\right.
$$


There is no quantum confinement in the $(x, y)$ plane. The quantized electron energies $E_{e z}\left(n_{e}\right)$ can be evaluated by solving the Schrödinger equation for the case of the confinement in the $z$ direction Zhao (1999)

$$
\left[-\frac{\hbar^{2}}{2 m_{e}} \frac{\partial^{2}}{\partial z^{2}}+V(z)\right] \Phi_{n, k}(z)=E_{n} \Phi_{n, k}(z)
$$

where

$$
E_{n}=E_{e z}\left(n_{e}\right)+E_{n 0}+\frac{(\hbar k)^{2}}{2 m_{e}}
$$

$V(z)$ is the confinement potential, and the electron wave function $\Psi_{n}(x, y, z)$ is given by Zhao (1999)

$$
\Psi_{n}(x, y, z)=u_{n}(x, y, z) \Phi_{n, k}(z) \frac{1}{\sqrt{L_{x} L_{y}}} \exp i\left(k_{x} x+k_{y} y\right)
$$

Here, $u_{n}(x, y, z)$ is the periodic Bloch function, $L_{x}$ and $L_{y}$ are the SOA dimensions in the $(x, y)$ directions, respectively. In the simplest case of a finite square-well potential $V_{0}$, the energy spectrum of QW is determined by the solutions of the following transcendental equations Brennan (1999).

$$
k_{1}=k_{2} \tan \left(\frac{k_{2} d_{z}}{2}\right) ; k_{1}=-k_{2} \cot \left(\frac{k_{2} d_{z}}{2}\right)
$$

where

$$
k_{1}=\sqrt{\frac{2 m_{e}\left(V_{0}-E\right)}{\hbar^{2}}} ; k_{2}=\sqrt{\frac{2 m_{e} E}{\hbar^{2}}} ;
$$

and $d_{z}$ is the QW dimension in the $z$ direction.

In this structure stimulated emission occurs only between discrete energy levels. QW-SOAs have superior performance as compared to bulk SOAs in terms of optical bandwidth and maximum output power. In SOAs, the saturation output power is inversely proportional to the differential gain coefficient, which is smaller in QW-SOAs.

The conventional SOA has rather poor carrier and photon confinement. Optical confinement can be enhanced by the implementation of a tapered graded index (GRIN) region on both sides of the well. A multi quantum well SOA (MQW-SOA) can be produced by stacking several layers of wells and barriers, usually created by molecular beam epitaxy (MBE) and/or metal organic vapor deposition (MOCVD) techniques. The optical gain of MQW is higher due to multiple QWs. Further improvement of the SOA performance is achieved by introducing an outer cladding region of a higher energy gap compared to the MQW barrier layers. The optical confinement factor of MQW SOAs increases approximately linearly with the number of QWs.

Many applications of SOA such as optical amplification, optical switching and signal processing require gain and phase shift insensitivity to the polarization. The polarization dependence of the phase shift is due to intrinsic or induced birefringence in SOA, so that the effective refraction index for the transverse electric (TE) mode is different from that of the transverse magnetic (TM) mode. These dependencies stem, in turn, from different quantization levels for heavy-hole $(\mathrm{HH})$ bands, which provide the TE-mode dominant optical gain, and light-hole (LH) bands, which provide the TM-mode dominant optical gain. The difference in the confinement factors for TE and TM modes leads to different TE and TM signal gains. 
The polarization sensitivity of MQW-SOAs can be significantly reduced when the active layer is strained, e.g. by introduction of lattice mismatch between the well and adjacent layers. In order to mitigate the polarization sensitivity problems in QW SOAs, several QW structures have been proposed such as low tensile-strained QWs, QWs with tensile barriers, tensile-strained QWs with compressive barriers, alternation of tensile and compressive QWs, and the delta-strained QW where strain is applied only at a shallow and highly strained layer called delta layer, that can yield a polarization-insensitive SOA at $\lambda=1550 \mathrm{~nm}$. In conventional QWs without delta layers, HH band energy levels are higher than those of LH bands, resulting in more TE gain relative to the TM mode. In delta-strained QWs, the delta layer causes a larger valence band discontinuity for $\mathrm{HH}$ bands than $\mathrm{LH}$ bands, so that the quantized energy levels of $\mathrm{HH}$ bands have a larger shift downward than those for the $\mathrm{LH}$ bands that shift upward. The delta layer pushes the LH band upward and the HH band downward such that their order is reversed.

In SOA applications requiring high optical power levels such as XGM, XPM and FWM, additional nonlinear birefringence is induced by the strong optical probe beam in such a way that the weak signal beam will undergo polarization dependent phase modulation. In some applications, however, this phenomenon can be utilized beneficially.

\subsection{QW SOA dynamics}

The dynamics of a QW heterostructure is usually described by a set of coupled rate equations containing injection current, injected carrier density, and photon density in the active region Zhao (1999). These equations provide an adequate tool for the analysis of XGM, XPM and FWM in QW SOA which is important for applications related to modern optical communications, especially in WDM architectures Reale (2001). Consider the carrier dynamics in a MQW SOA during the propagation of Gaussian pulses with the duration of several ps. The main transport mechanisms across the active region are the exchange of carriers between different QWs, exchange between a QW and separate confinement heterolayers $(\mathrm{SCH})$, and the carrier injection from the $\mathrm{SCH}$. In such a model each QW interacts with the neighboring QWs and with the surrounding SCHs Reale (2001). The rate equations in such a case differ significantly from the bulk SOA equations (10), (11) due to the number $M$ of QWs. These rate equations have the form Reale (2001)

$$
\begin{gathered}
\frac{\partial S}{\partial z}+\frac{1}{v_{g}} \frac{\partial S}{\partial t}=\sum_{i=1}^{M} g\left(N_{i}\right) S_{i}-\frac{1}{v_{g}} \frac{S}{\tau_{p}}+\frac{1}{v_{g}} \sum_{i=1}^{M} \beta R_{\text {spon }}\left(N_{i}\right) \\
\frac{\partial N_{i}}{\partial t}=\eta_{i n j} \frac{I_{i n j}}{q L_{i}}-R\left(N_{i}\right)-R_{s t, i}
\end{gathered}
$$

where $S(z, t)=\sum_{i=1}^{M} S_{i}$ is the total photon concentration, $S_{i}$ is the photon density generated in $i$ th QW, $N_{i}(z, t)$ is the carrier density in $i$ th $\mathrm{QW}, g\left(N_{i}\right)$ is the gain-carrier density relationship, $\tau_{p}$ is the photon lifetime, $\beta$ is the coupling factor of the spontaneous emission $R_{\text {spon }}\left(N_{i}\right)$, $\eta_{i n j}$ is the injection efficiency, $I_{i n j}$ is the injection current, $R\left(N_{i}\right)$ is the recombination term accounting for trap-related, spontaneous, and Auger recombinations, $L_{i}$ is the thickness of the particular layer considered, and $R_{s t, i}$ accounts for stimulated recombination. The polarization dependence of the gain for TE and TM modes is neglected. The typical values of the MQW SOA main parameters are: $M=10$, the QW width $L_{w}=48 \AA$, device length $L=700 \mu m, \beta=$ $10^{-4}$, the threshold density $N_{t h}=1.5 \times 10^{18} \mathrm{~cm}^{-3}, v_{g}=8.5 \times 10^{9} \mathrm{~cm} / \mathrm{s}$, optical confinement 
factor 0.02, the losses in the active region $\alpha_{l}=\left(v_{g} \tau_{p}\right)^{-1} \sim 10 \mathrm{~cm}^{-1}$, the bias currents $I_{i n j} \sim$ $50-150 \mathrm{~m} A$, the gain for the probe light at $\lambda=1560 \mathrm{~nm}$ is $17-21 \mathrm{~dB}$ Reale (2001).

We introduce a delayed time reference $t=t^{\prime}-z / v_{g}$ and rewrite equation (29) as

$$
\frac{\partial S}{\partial z}=g(N)-\frac{1}{v_{g}} \frac{S}{\tau_{p}}+\frac{1}{v_{g}} \beta R_{\text {spon }}
$$

which yields a formal solution

$$
S_{\text {out }}=S_{\text {in }} \exp \left[\int_{0}^{L}\left(g-\alpha_{l}+\frac{1}{v_{g}} \beta R_{\text {spon }}\right) d z\right]
$$

Equations (30) and (31) have been solved numerically and showed a good accord with the experimental data for picosecond optical pulse propagation through a MQW SOA Reale (2001). Typically, the ultrafast pulse is simultaneously amplified and undergoes shape modification for high bias currents of about $150 \mathrm{~mA}$. The shape evolution is mainly due to the gain saturation in the MQW SOA. As a result, the total gain for a single optical pulse reduces from $20 \mathrm{~dB}$ at the lowest energy to $1 \mathrm{~dB}$ at the highest energy demonstrating strong nonlinearity of MQW SOAs Reale (2001).

\subsection{QD structure and energy spectrum}

Quantization of electron states in all three dimensions results in creation of a novel physical object - a macroatom, or quantum dot (QD) containing a zero dimensional electron gas. Size quantization is effective when the QD's three dimensions are of the same order of magnitude as the electron de Broglie wavelength which is about several nanometers Ustinov (2003). QD is a nanomaterial confined in all the three dimensions, and for this reason it has unique electronic and optical properties that do not exist in bulk semiconductor material Ohtsu (2008). An electron-hole pair created by light in a QD has discrete energy eigenvalues due to carrier confinement. This phenomenon is called a quantum confinement effect Ohtsu (2008).

There exist different types of QDs based on different technologies that operate in different parts of spectrum such as $\operatorname{In}(\mathrm{Ga})$ As QDs grown on GaAs substrates, InAs QDs grown on InP substrates, and colloidal free-standing InAs QDs. QD structures are commonly realized by a self-organized epitaxial growth where QDs are statistically distributed in size and area. A widely used QD fabrication method is a direct synthesis of semiconductor nanostructures based on island formation during strained-layer heteroepitaxy called the Stranski-Krastanow (SK) growth mode Ustinov (2003). Spontaneously growing QDs are said to be self-assembling. SK growth has been investigated intensively for InAs on GaAs, InP on GaInP, and Ge on Si structures Ustinov (2003). The energy shift of the emitted light is determined by the size of QDs that can be adjusted within a certain range by changing the amount of deposited QD material. Evidently, smaller QDs emit photons of shorter wavelengths Ustinov (2003). The main advantages of the SK growth are Ustinov (2003):

1. SK growth permits the preparation of extremely small QDs in a maskless process without lithography and etching which makes it a promising technique to realize QD lasers.

2. A great number of QDs is formed in one simple deposition step.

3. Synthesized QDs are highly uniform in size and composition.

4. QDs can be covered epitaxially by a host material without any crystal or interface defects. 
The simplest QD models are described by the spherical boundary conditions for an electron or a hole confined in a spherical QD with radius $R$, or by the cubic boundary conditions for a parallelepiped QD with side length $L_{x, y, z}$ Ohtsu (2008). In the first case, the electron and hole energy spectra $E_{e, n l m}$ and $E_{h, n l m}$ are respectively given by Ohtsu (2008)

$$
E_{e, n l m}=E_{g}+\frac{\hbar^{2}}{2 m_{e}}\left(\frac{\alpha_{n l}}{R}\right)^{2} ; E_{h, n l m}=\frac{\hbar^{2}}{2 m_{h}}\left(\frac{\alpha_{n l}}{R}\right)^{2}
$$

where

$$
n=1,2,3, \ldots ; l=0,1,2, \ldots n-1 ; m=0, \pm 1, \pm 2, . . \pm l,
$$

$E_{g}$ is the QD semiconductor material band gap, $m_{e, h}$ are the electron and hole effective mass, respectively, and $\alpha_{n l}$ is the $n$-th root of the spherical Bessel function of order $l$. In the second case, the energy eigenvalues $E_{e, n l m}$ and $E_{h, n l m}$ are respectively given by Ohtsu (2008)

$$
\begin{gathered}
E_{e, n l m}=E_{g}+\frac{\hbar^{2} \pi^{2}}{2 m_{e}}\left[\left(\frac{n}{L_{x}}\right)^{2}+\left(\frac{l}{L_{y}}\right)^{2}+\left(\frac{m}{L_{z}}\right)^{2}\right] \\
E_{h, n l m}=\frac{\hbar^{2} \pi^{2}}{2 m_{h}}\left[\left(\frac{n}{L_{x}}\right)^{2}+\left(\frac{l}{L_{y}}\right)^{2}+\left(\frac{m}{L_{z}}\right)^{2}\right]
\end{gathered}
$$

The DOS function $\rho_{Q D}(E)$ for an array of QDs has the form Ustinov (2003)

$$
\rho_{Q D}(E)=\sum_{n} \sum_{m} \sum_{l} 2 n_{Q D} \delta\left(E-E_{e, n l m}\right)
$$

where $\delta\left(E-E_{e, n l m}\right)$ is the $\delta$-function, and $n_{Q D}$ is the surface density of QDs. The active layer with QDs and the structure of QD conduction band are shown in Fig. 2.

Detailed theoretical and experimental investigations of InAs/GaAs and InAs QDs electronic structure taking into account their realistic lens, or pyramidal shape, size, composition profile, and production technique (SK, colloidal) have been carried out Bimberg (1999), Bányai (2005), Ustinov (2003). A system of QDs can be approximated with a three energy level model in the conduction band containing a spin degenerate ground state (GS), fourfold degenerate excited state (ES) with comparatively large energy separations of about $50-70 \mathrm{meV}$, and a narrow continuum wetting layer (WL). The electron WL is situated $150 \mathrm{meV}$ above the lowest electron energy level in the conduction band, i.e. GS and has a width of approximately $120 \mathrm{meV}$. In real cases, QDs vary in size, shape, and local strain which leads to fluctuations in the quantized energy levels and inhomogeneous broadening in the optical transition energy. Gaussian distribution may be used for description of the QD sizes Bányai (2005). The QDs and WL are surrounded by a barrier material which prevents direct coupling between QD layers. The absolute number of states in the WL is much larger than in the QDs. GS and ES in QDs are characterized by homogeneous and inhomogeneous broadening Bányai (2005). The homogeneous broadening caused by scattering of the optically generated electrons and holes with imperfections, impurities, phonons, or through the radiative electron-hole pair recombination Bányai (2005) is about $15 \mathrm{meV}$ at room temperature.

The eigenspectrum of a single QD fully quantized in three dimensions consists of a discrete set of eigenvalues depending only on the number of atoms in it. Variations of eigenenergies from QD to QD are caused by variations of QD's strain and shape. The finite carrier lifetime results in Lorentzian broadening of a finite width Ustinov (2003). The optical spectrum of 


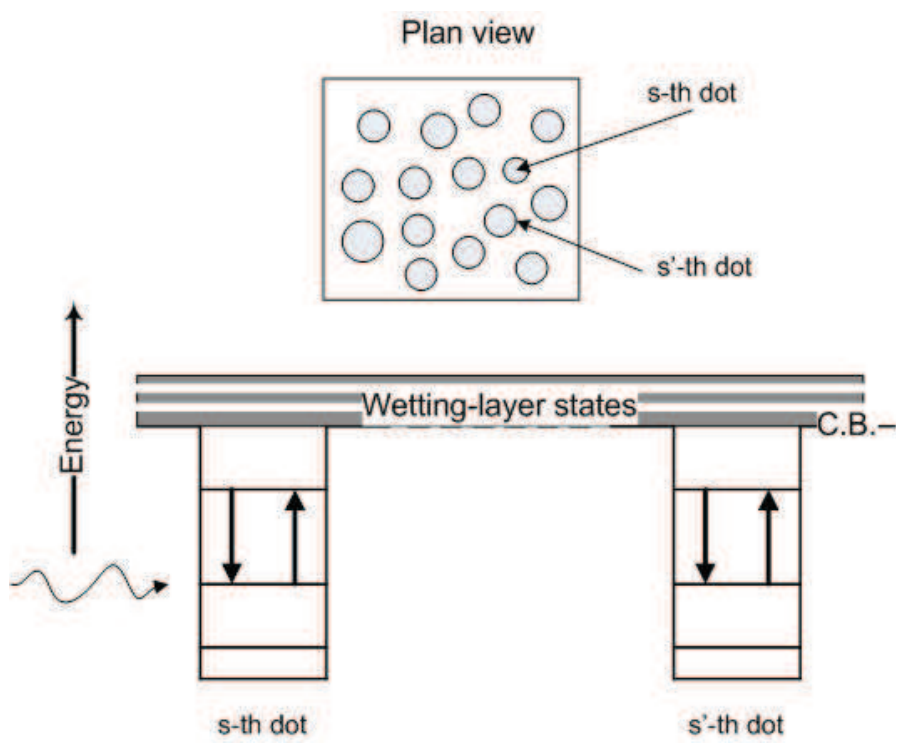

Fig. 2. Plan view of active layer with QDs (above); the QD conduction band structure (below)

QDs consists of a series of transitions between the zero-dimensional electron gas energy states where the selection rules are determined by the form and symmetry of QDs Ustinov (2003). In(Ga)As/GaAs QDs are characterized by emission at wavelengths no longer than $\lambda=1.35 \mu \mathrm{m}$, while the InAs/InP QDs have been proposed for emission at the usual telecommunication wavelength $\lambda=1.55 \mu m$ Ustinov (2003).

\subsection{Structure and operation of QD SOA}

In this section, we will discuss the structure and operation principles of QD SOA. Theory of QD SOA operation based on the electron rate equations and photon propagation equation has been developed in a large number of works Berg (2004), Qasaimeh (2003), Qasaimeh (2004), Ben Ezra (September 2005), Ben Ezra (October 2005), Ben Ezra (2007).

The active region of a QD SOA is a layer including self-assembled InGaAs QDs on a GaAs substrate Sugawara (2004). Typically, the QD density per unit area is about $\left(10^{10}-10^{11}\right) \mathrm{cm}^{-2}$. The bias current is injected into the active layer including QDs, and the input optical signals are amplified via the stimulated emission or processed via the optical nonlinearity by QDs Sugawara (2004). The stimulated radiative transitions occur between GS and the valence band of QDs. A detailed theory of QD SOAs based on the density matrix approach has been developed in the pioneering work of Sugawara (2004) where the linear and nonlinear optical responses of QD SOAs with arbitrary spectral and spatial distribution of quantum dots in active region under the multimode light propagation have been considered. It has been shown theoretically that XGM takes place due to the coherent terms under the condition that the mode separation is comparable to or less than the polarization relaxation rate $\left|\omega_{m}-\omega_{n}\right| \leq \Gamma_{g}$ where $\omega_{m, n}$ are the mode frequencies and the relaxation time $\tau=\Gamma_{g}^{-1}=130 \mathrm{fs}$ Sugawara (2004). XGM is also possible in the case of the incoherent nonlinear polarization, or the so-called incoherent spectral hole burning Sugawara (2004). It has been assumed that XGM occurred only for signals with a detuning limited by 
the comparatively small homogeneous broadening, and for this reason the ensemble of QDs should be divided into groups by their resonant frequency of the GS transition between the conduction and valence bands Sugawara (2004).

The phenomenological approach to the QD SOA dynamics is based on the rate equations for the electron densities of GS, ES and for combined WL and barrier serving as a reservoir. It is determined by electrons, because of the much larger effective mass of holes and their smaller state spacing Berg (2004).

In the QD SOA-MZI, optical signals propagate in an active medium with a gain determined by the rate equations for the electron transitions in QD-SOA between WL, GS and ES Qasaimeh (2003), Qasaimeh (2004), Ben Ezra (2008), Ben Ezra (September 2005). Unlike this model Sun (2005), we have taken into account two energy levels in the conduction band: GS and ES. The stimulated and spontaneous radiative transitions occur from GS to the QD valence band level. The system of the rate equations accounts for the following transitions:

1. the fast electron transitions from WL to ES with the relaxation time $\tau_{w 2} \sim 3 p s$;

2. the fast electron transitions between ES and GS with the relaxation time from ES to GS $\tau_{21}=0.16 p s$ and the relaxation time from GS to ES $\tau_{12} \sim 1.2 p s ;$

3. the slow electron escape transitions from ES back to WL with the electron escape time $\tau_{2 w} \sim 1 n s$.

The balance between the WL and ES is determined by the shorter time $\tau_{w 2}$ of QDs filling. Carriers relax quickly from the ES level to the GS level, while the former serves as a carrier reservoir for the latter Berg (2001). In general case, the radiative relaxation times depend on the bias current. However, it can be shown that for moderate values of the WL carrier density $N_{w} \sim\left(10^{14}-10^{15}\right) \mathrm{cm}^{-3}$ this dependence can be neglected Berg (2001), Berg (November 2004).

The spontaneous radiative time $\tau_{1 R} \gtrsim(0.4-0.5) n s$ in QDs remains large enough Sakamoto (2000), Qasaimeh (2003), Sugawara (2004), Qasaimeh (2004), Matthews (2005). The carrier dynamics is characterized by slow relaxation processes between WL and ES, and the rapidly varying coherent nonlinear population terms vanish after averaging over the comparatively large relaxation time $\tau_{w 22} \sim$ several ps from the two-dimensional WL to the ES. We have taken into account only incoherent population terms because for XGM between modes with the maximum detuning $\Delta \lambda_{\max }=30 \mathrm{~nm}$, within the especially important in optical communications conventional band (C-band) of $\lambda=(1530 \div 1565) \mathrm{nm}$, the condition $\omega_{1}-$ $\omega_{2}>\Gamma_{g}^{-1}$ is valid even for the lowest relaxation time from the ES to GS $\tau_{21}=0.16 p s$, and the rapidly varying coherent beating terms are insignificant Sugawara (2004). The direct carrier capture into the GS is neglected due to the fast intradot carrier relaxation and the large energy separation between GS and WL and it is assumed that the charge neutrality condition in GS is valid. The rate equations account for both fast transitions form WL to ES and GS and slow dynamics of the spontaneous transitions and electron escape from ES back to WL Qasaimeh (2003), Qasaimeh (2004), Ben Ezra (2007). They have the form Qasaimeh (2003), Qasaimeh (2004), Ben Ezra (2007).

$$
\begin{gathered}
\frac{\partial N_{w}}{\partial t}=\frac{J}{e L_{w}}-\frac{N_{w}(1-h)}{\tau_{w 2}}+\frac{N_{w} h}{\tau_{2 w}}-\frac{N_{w}}{\tau_{w R}}, \\
\frac{\partial h}{\partial t}=\frac{N_{w} L_{w}(1-h)}{N_{Q} \tau_{w 2}}-\frac{N_{w} L_{w} h}{N_{Q} \tau_{2 w}}-\frac{(1-f) h}{\tau_{21}}+\frac{f(1-h)}{\tau_{12}},
\end{gathered}
$$




$$
\begin{gathered}
\frac{\partial f}{\partial t}=\frac{(1-f) h}{\tau_{21}}-\frac{f(1-h)}{\tau_{12}}-\frac{f^{2}}{\tau_{1 R}} \\
-\frac{g_{p} L}{N_{Q}}(2 f-1) S_{p} \frac{c}{\sqrt{\varepsilon_{r}}}-\frac{g_{s} L}{N_{Q}}(2 f-1) S_{s} \frac{c}{\sqrt{\varepsilon_{r}}} .
\end{gathered}
$$

Here, $S_{p}, S_{s}$ are the CW pump and on-off-keying (OOK) modulated signal wave photon densities, respectively, $L$ is the length of SOA, $g_{p}, g_{s}$ are the pump and signal wave modal gains, respectively, $f$ is the electron occupation probability of GS, $h$ is the electron occupation probability of ES, $e$ is the electron charge, $\tau_{2 w}$ is the electron escape time from the ES to the $W L, \tau_{w R}$ is the spontaneous radiative lifetime in $W L, \tau_{1 R}$ is the spontaneous radiative lifetime in QDs, $N_{Q}$ is the surface density of QDs, $N_{w}$ is the electron density in the WL, $L_{w}$ is the effective thickness of the active layer, $\tau_{21}$ is the electron relaxation time from the ES to GS and $\tau_{12}$ is the electron relaxation time from the GS to the ES, and $\varepsilon_{r}$ is the SOA material permittivity. The modal gain $g_{p, s}(\omega)$ is given by Uskov (2004)

$$
g_{p, S}(\omega)=\frac{2 \Gamma N_{Q}}{a} \int d \omega F(\omega) \sigma\left(\omega_{0}\right)(2 f-1)
$$

where the number $l$ of QD layers is assumed to be $l=1$, the confinement factor $\Gamma$ is assumed to be the same for both the signal and the pump waves, $a$ is the mean size of QDs, $\sigma\left(\omega_{0}\right)$ is the cross section of interaction of photons of frequency $\omega_{0}$ with carriers in QD at the transition frequency $\omega$ including the homogeneous broadening factor, $F(\omega)$ is the distribution of the transition frequency in the QD ensemble which is assumed to be Gaussian Qasaimeh (2004), Uskov (2004). It is related to the inhomogeneous broadening and it is described by the expression Uskov (2004)

$$
F(\omega)=\frac{1}{\Delta \omega \sqrt{\pi}} \exp \left[-\frac{(\omega-\bar{\omega})^{2}}{(\Delta \omega)^{2}}\right]
$$

where the parameter $\Delta \omega$ is related to the inhomogeneous linewidth $\gamma_{\text {in hom }}=2 \sqrt{\ln 2} \Delta \omega$, and $\bar{\omega}$ is the average transition frequency.

In order to describe adequately XGM and XPM in QD SOA we should take into account the interaction of QDs with optical signals. The optical signal propagation in a QD SOA is described by the following truncated equations for the slowly varying $\mathrm{CW}$ and pulse signals photon densities and phases $S_{C W, P}=P_{C W, P} /\left(\hbar \omega_{C W, P}\left(v_{g}\right)_{C W, P} A_{e f f}\right)$ and $\theta_{C W, P}$ Agrawal (1989).

$$
\begin{gathered}
\frac{\partial S_{C W, P}(z, \tau)}{\partial z}=\left(g_{C W, P}-\alpha_{i n t}\right) S_{C W, P}(z, \tau) \\
\frac{\partial \theta_{C W, P}}{\partial z}=-\frac{\alpha}{2} g_{C W, P}
\end{gathered}
$$

Here $P_{C W, P}$ are the $C W$ and pulse signal optical powers, respectively, $A_{\text {eff }}$ is the QD SOA effective cross-section, $\omega_{C W, P},\left(v_{g}\right)_{C W, P}$ are the $C W$ and pulse signal group angular frequencies and velocities, respectively, $g_{C W, P}$ are the active medium (SOA) gains at the corresponding optical frequencies, and $\alpha_{i n t}$ is the absorption coefficient of the SOA material. For the pulse propagation analysis, we replace the variables $(z, t)$ with the retarded frame variables $\left(z, \tau=t \mp z / v_{g}\right)$. For optical pulses with a duration $T \gtrsim 10 p$ s the optical radiation 
of the pulse fills the entire active region of a QD SOA of length $L \lesssim 1 \mathrm{~mm}$ and the propagation effects can be neglected Gehrig (2002). Hence, in our case the photon densities

$$
S_{C W, P}(z, \tau)=\left(S_{C W, P}(\tau)\right)_{\text {in }} \exp \left[\int_{0}^{z}\left(g_{C W, P}-\alpha_{i n t}\right) d z^{\prime}\right]
$$

can be averaged over the QD SOA length $L$ which yields

$$
S_{C W, P}(\tau)=\frac{1}{L}\left(S_{C W, P}(\tau)\right)_{i n} \int_{0}^{L} d z \exp \left[\int_{0}^{z}\left(g_{C W, P}-\alpha_{i n t}\right) d z^{\prime}\right]
$$

Solution of equation (44) yields for the phases which should be inserted into MZI equation (53)

$$
\theta_{C W, P}(\tau)=-(\alpha / 2) \int_{0}^{L} d z g_{C W, P}
$$

The time-dependent variations of the carrier distributions in the QDs and WL result in strong phase changes (44) during the light propagation in the QD SOA Gehrig (2002). System of equations (38)-(40) with the average pump and signal photon densities (46) and phases (47) constitutes a complete set of equations describing XGM and XPM in QD SOA related by the LEF $\alpha$ as it is seen from equations (43), (44) and (47). The possibility of XGM in QD SOAs due to the connections between different QDs through WL at detunings between a signal and a pumping larger than the homogeneous broadening has been thoroughly investigated theoretically Ben Ezra (2007).

The advantages of QD SOAs as compared to bulk SOAs are the ultrafast gain recovery of about a few picoseconds, broadband gain, low NF, high saturation output power and high FWM efficiency Akiyama (2007). For instance, distortion free output power of $23 \mathrm{dBm}$ has been realized which is the highest among all the SOAs Akiyama (2007). A gain of $>25 d B$, $N F$ of $<5 d B$ and output saturation power of $>20 \mathrm{dBm}$ can be obtained simultaneously in the wavelength range of 90 $\mathrm{nm}$ Akiyama (2007).

\section{Recent advances in SOA applications}

\subsection{All-optical pulse generation}

Ultra wideband (UWB) communication is a fast emerging technology that offers new opportunities such as high data rates, low equipment cost, low power, precise positioning capability and extremely low signal interference. A contiguous bandwidth of $7.5 \mathrm{GHz}$ is available in the frequency interval of $(3.1-10.6) \mathrm{GHz}$ at an extremely low maximum power output of $-41.3 \mathrm{dBm} / \mathrm{MHz}$ limited by the regulations of Federal Communication Commission (FCC) Ghawami (2005). Impulse radio (IR) UWB communication technique is a carrier free modulation using very narrow radio frequency (RF) pulses generated by UWB pulse generators Yao (2007). However, high data rate UWB systems are limited to distances less than $10 m$ due the constraints on allowed emission levels Yao (2007), Ran (2009). In order to increase IR UWB transmission distances, a new concept based on UWB technologies and the optical fiber technology has been proposed that is called UWB radio over optical fibre (UROOF) Ran (2009). The IR UWB signals of several GHz are superimposed on the optical continuous wave (CW) carrier and transmitted transparently over an optical fiber Ran (2009), Yao (2007). The 
UROOF technology permits to avoid the high cost additional electronic components required for signal processing and enables the integration of all RF and optical transmitter/receiver components on a single chip.

In order to distribute UWB signals via optical fibers, it is desirable to generate these signals directly in the optical domain. The advantages of the all-optical methods are following: decreasing of interference between electrical devices, low loss and light weight of optical fibers Lin (2005), Yao (2007), Wang (2006).

Typically, Gaussian waveforms are used in UWB communications due to their simplicity, achievability, and almost uniform distribution over their frequency spectrum Yao (2007), Ghawami (2005). The basic Gaussian pulse $y_{g 1}$, a Gaussian monocycle $y_{g 2}$ and a Gaussian doublet $y_{g 3}$ are given by Ghawami (2005).

$$
\begin{gathered}
y_{g 1}=K_{1} \exp \left(-\frac{t^{2}}{\tau^{2}}\right) ; \\
y_{g 2}=K_{2}\left(-\frac{2 t}{\tau^{2}}\right) \exp \left(-\frac{t^{2}}{\tau^{2}}\right) ; y_{g 3}=K_{3}\left(-\frac{2}{\tau^{2}}\right)\left(1-\frac{2 t^{2}}{\tau^{2}}\right) \exp \left(-\frac{t^{2}}{\tau^{2}}\right)
\end{gathered}
$$

where $\tau$ is the time-scaling factor, and $K_{1,2,3}$ are the normalization constants:

$$
K_{1}=\sqrt{\frac{E_{1}}{\tau \sqrt{\pi / 2}}} ; K_{2}=\sqrt{\frac{\tau E_{2}}{\sqrt{\pi / 2}}} ; K_{3}=\tau \sqrt{\frac{\tau E_{3}}{3 \sqrt{\pi / 2}}}
$$

There exist three main optical IR UWB generation techniques Yao (2007)

1. UWB pulse generation based on phase-modulation-to-intensity-modulation (PM-IM) conversion.

2. UWB pulse generation based on a photonic microwave delay line using SOA.

3. UWB pulse generation based on optical spectral shaping and dispersion-induced frequency-to-time mapping. All-optical methods of UWB pulse generation are based on nonlinear optical processes in SOA such as XPM and XGM.

We concentrate on the all-optical methods of UWB pulse generation based on XPM and XGM in SOA. Consider first the method based on XPM. A probe CW signal generated by CW laser diode and a light wave modulated by the Mach-Zehnder modulator (MZM) are simultaneously fed into SOA, the probe signal will undergo both XGM and XPM, and the phase $\Phi_{c}$ of the output signal varies approximately proportionally to Gaussian pulse train power $P_{S}(t)$ Dong (2009)

$$
\Phi_{c}=K P_{S}(t)+\Phi_{0}
$$

where $K$ is the proportionality constant and $\Phi_{0}$ is the initial phase. The chirp $\Delta v_{c}(t)$ of the probe signal is the first order derivative of the phase given by Dong (2009)

$$
\Delta v_{c}(t)=-\frac{1}{2 \pi} \frac{d \Phi_{c}}{d t}=-\frac{K}{2 \pi} \frac{d P_{s}(t)}{d t}
$$

The chirp (52) is a monocycle, according to definition (49). Its value may be positive or negative. UWB doublet pulses can be obtained by combining positive and negative monocycles with a proper delay Dong (2009). The shortages of the proposed method are the necessity for complicated electronic circuit for generation short electric Gaussian pulses, the 
use of an electro-optic phase modulator (EOM), the need for a comparatively long singlemode fiber (SMF), and a comparatively low operation rate and high bias currents of bulk SOAs.

Recently, the theory of a novel all-optical method of the IR UWB pulse generation has been proposed Ben Ezra (2008). QD SOA can be inserted into one arm of an integrated Mach-Zehnder interferometer (MZI) which results in an intensity dependent optical signal interference at the output of MZI Ben Ezra (2008). The IR UWB pulse generation process is based both on XPM and XGM in QD SOA characterized by an extremely high optical nonlinearity, low bias current, and high operation rate Sugawara (2004). Unlike other proposed all-optical methods, we need no optical fibers, FBG and EOM substantially reducing the cost and complexity of the IR UWB generator. The IR UWB signals generated by the proposed QD SOA based MZI structure have the form of the Gaussian doublet. The shape of the signal and its spectrum can be tailor-made for different applications by changing the QD SOA bias current and optical power. The diagram of the MZI with QD SOA is shown in Fig. 3.

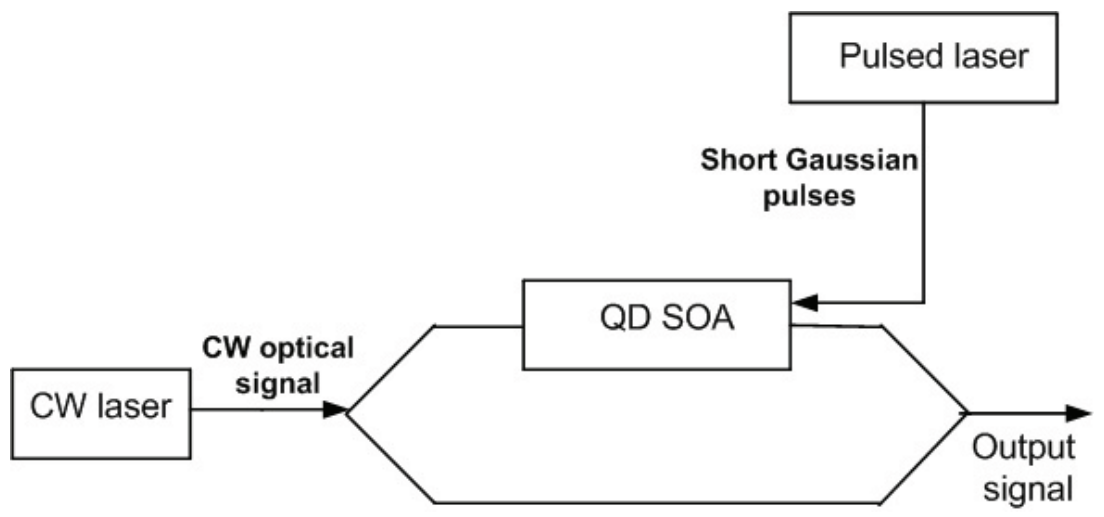

Fig. 3. MZI with QD SOA in the upper arm

The pulsed laser produces a train of short Gaussian pulses counter-propagating with respect to the input CW optical signal. The CW signal propagating through the upper arm of MZI transforms into the Gaussian pulse at the output of the MZI due to XPM and XGM with the train of Gaussian pulses. The optical signal in the linear lower arm of MZI remains CW, and the phase shift $\phi_{2}=$ const in the lower arm of MZI is constant. Both these pulses interfere at the output of MZI, and the output pulse shape is defined by the power dependent phase difference $\Delta \phi(t)=\phi_{1}(t)-\phi_{2}(t)$ where $\phi_{1,2}(t)$ are the phase shifts in the upper and lower arms of MZI, respectively. The MZI output optical power $P_{\text {out }}$ is given by Wang (2004).

$$
P_{\text {out }}=\frac{P_{0}}{4}\left[G_{1}(t)+G_{2}(t)-2 \sqrt{G_{1}(t) G_{2}(t)} \cos \Delta \phi(t)\right]
$$

where $G_{1,2}(t)=\exp \left(g_{1,2} L_{1,2}\right), g_{1,2}, L_{1,2}$ are the amplification factors of the upper and lower arms of MZI, the time-dependent gain, the SOA gain, and the active medium length, respectively. The relation between the MZI phase shift and its amplification factor is given by $\Delta \phi(t)=-\left(\alpha_{L} / 2\right) \ln G_{1}(t)$. The shape of the output pulse is determined by the time dependence of $G_{1}(t)$ both directly and through $\Delta \phi(t)$ according to equation (53) resulting in a Gaussian doublet under certain conditions determined by the QD SOA dynamics. 


\subsection{All-optical signal processing}

Recently, theoretical model of an ultra-fast all-optical signal processor based on the QD SOA-MZI where XOR operation, WC, and 3R signal regeneration can be simultaneously carried out by AO-XOR logic gates for bit rates up to $(100-200) \mathrm{Gb} / \mathrm{s}$ depending on the value of the bias current $I \sim(30-50) m A$ has been proposed. Ben Ezra (2009). The structure of the proposed processor is shown in Fig. 4.

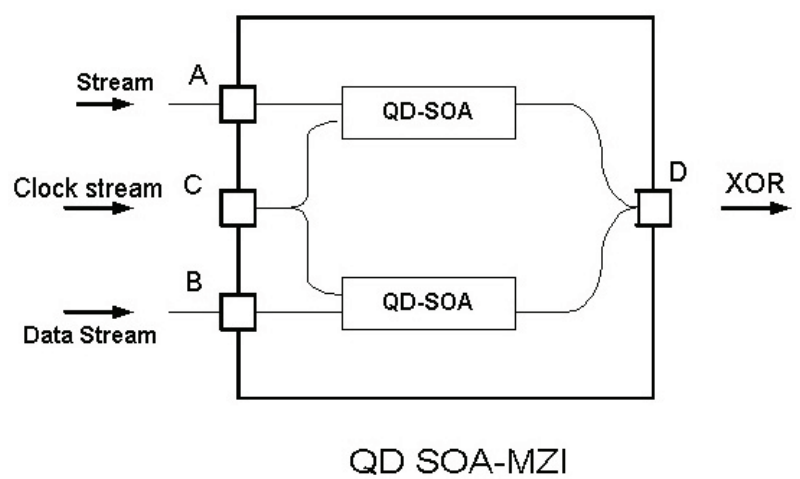

Fig. 4. The structure of the ultra-fast all-optical signal processor based on QD SOA-MZI

The theoretical analysis of the proposed ultra-fast QD SOA-MZI processor is based on combination of the MZI model with the QD-SOA nonlinear characteristics and the dynamics. At the output of MZI, the CW optical signals from the two QD SOAs interfere giving the output intensity are determined by equation (53) with the CW or the clock stream optical signal power $P_{\text {in }}$ instead of $P_{0}$ Sun (2005), Wang (2004). When the control signals $A$ and/or $B$ are fed into the two SOAs they modulate the gain of the SOAs and give rise to the phase modulation of the co-propagating CW signal due to LEF $\alpha_{L}$ Agrawal (2001), Agrawal (2002), Newell (1999). LEF values may vary in a large interval from the experimentally measured value of LEF $\alpha_{L}=0.1$ in InAs QD lasers near the gain saturation regime Newell (1999) up to the giant values of LEF as high as $\alpha_{L}=60$ measured in InAs/InGaAs QD lasers Dagens (2005). However, such limiting cases can be achieved for specific electronic band structure Newell (1999), Dagens (2005), Sun (2004). The typical values of LEF in QD lasers are $\alpha_{L} \approx(2-7)$ Sun (2005). Detailed measurements of the LEF dependence on injection current, photon energy, and temperature in QD SOAs have also been carried out Schneider (2004). For low-injection currents, the LEF of the dot GS transition is between 0.4 and 1 increasing up to about 10 with the increase of the carrier density at room temperature Schneider (2004). The phase shift at the QD SOA-MZI output is given by Wang (2004)

$$
\phi_{1}(t)-\phi_{2}(t)=-\frac{\alpha_{L}}{2} \ln \left(\frac{G_{1}(t)}{G_{2}(t)}\right)
$$

It is seen from equation (54) that the phase shift $\phi_{1}(t)-\phi_{2}(t)$ is determined by both LEF and the gain. For the typical values of LEF $\alpha_{L} \approx(2-7)$, gain $g_{1,2}=11.5 \mathrm{~cm}^{-1}, L_{1,2}=1500 \mu \mathrm{m}$ the phase shift of about $\pi$ is feasible. 


\subsection{All-optical logics}

Consider an AO-XOR gate based on integrated SOA-MZI which consists of a symmetrical MZI where one QD SOA is located in each arm of the interferometer Sun (2005). Two optical control beams $A$ and $B$ at the same wavelength $\lambda$ are inserted into ports $A$ and $B$ of MZI separately. A third signal, which represents a clock stream of continuous series of unit pulses is split into two equal parts and injected into the two SOAs. The detuning $\Delta \omega$ between the signals $A, B$ and the third signal should be less than the homogeneous broadening of QDs spectrum. In such a case the ultrafast operation occurs. In the opposite case of a sufficiently large detuning comparable with the inhomogeneous broadening, XGM in a QD SOA is also possible due to the interaction of QDs groups with essentially different resonance frequencies through WL for optical pulse bit rates up to $10 \mathrm{~Gb} / \mathrm{s}$ Ben Ezra (September 2005). When $A=B=0$, the signal at port $C$ traveling through the two arms of the SOA acquires a phase difference of $\pi$ when it recombines at the output port $D$, and the output is " 0 " due to the destructive interference. When $A=1, B=0$, the signal traveling through the arm with signal $A$ acquires a phase change due to XPM between the pulse train $A$ and the signal. The signal traveling through the lower arm does not have this additional phase change which results in an output " 1 " Sun (2005). The same result occurs when $A=0, B=1$ Sun (2005). When $A=1$ and $B=1$ the phase changes for the signal traveling through both arms are equal, and the output is " 0 ".

\subsection{Wavelength conversion}

An ideal wavelength convertor (WC) should have the following properties: transparency to bit rates and signal formats, fast setup time of output wavelength, conversion to both shorter and longer wavelengths, moderate input power levels, possibility for no conversion regime, insensitivity to input signal polarization, low-chirp output signal with high extinction ratio and large signal-to-noise ratio (SNR), and simple implementation Ramamurthy (2001). Most of these requirements can be met by using SOA. The XGM method using SOAs is especially attractive due to its simple realization scheme for WC Agrawal (2001). However, the main disadvantages of this method are substantial phase distortions due to frequency chirping, degradation due to spontaneous emission, and a relatively low extinction ratio Agrawal (2001). These parameters may be improved by using QD-SOAs instead of bulk SOAs due to pattern-effect-free high-speed WC of optical signals by XGM, a low threshold current density, a high material gain, high saturation power, broad gain bandwidth, and a weak temperature dependence as compared to bulk and MQW devices Ustinov (2003). We combine the advantages of QD-SOAs as a nonlinear component and MZI as a system whose output signal can be easily controlled. In the situation where one of the propagating signals $A$ or $B$ is absent, the CW signal with the desired output wavelength is split asymmetrically to each arm of MZI and interferes at the output either constructively or destructively with the intensity modulated input signal at another wavelength. The state of interference depends on the relative phase difference between the two MZI arms which is determined by the SOAs. In such a case the QD SOA-MZI operates as an amplifier of the remaining propagating signal. Then, the operation with the output "1" may be characterized as a kind of WC due to XGM between the input signal $A$ or $B$ and the clock stream signal. The possibility of the pattern-effect-free WC by XGM in QD SOAs has been demonstrated experimentally at the wavelength of $1.3 \mu \mathrm{m}$ Sugawara (2004). 


\subsection{3 $3 \mathrm{R}$ regeneration}

Short optical pulses propagating in optical fibers are distorted due to the fiber losses caused by material absorption, Rayleigh scattering, fiber bending, and broadening caused by the material dispersion, waveguide dispersion, polarization-mode dispersion, intermodal dispersion Agrawal (2001), Agrawal (2002). 3R regeneration is essential for successful logic operations because of the ultra-fast data signal distortions. 3R regeneration requires an optical clock and a suitable architecture of the regenerator in order to perform a clocked decision function Sartorius (2001). In such a case, the shape of the regenerated pulses is defined by the shape of the clock pulses Sartorius (2001).

The proposed QD SOA-MZI ultra-fast all-optical processor can successfully solve three problems of $3 R$ regeneration. Indeed, the efficient pattern-effect free optical signal re-amplification may be carried out in each arm by QD-SOAs. WC based on an all-optical logic gate provides the re-shaping since noise cannot close the gate, and only the data signals have enough power to close the gate Sartorius (2001). The re-timing in QD-SOA-MZI based processor is provided by the optical clock which is also essential for the re-shaping Sartorius (2001). Hence, if the CW signal is replaced with the clock stream, the 3R regeneration can be carried out simultaneously with logic operations. The analysis shows that for strongly distorted data signals a separate processor is needed providing $3 \mathrm{R}$ regeneration before the data signal input to the logic gate.

\subsection{Slow light propagation in SOA}

One of the challenges of the optoelectronic technology is the ability to store an optical signal in optical format. Such an ability can significantly improve the routing process by reducing the routing delay, introducing data transparency for secure communications, and reducing the power and size of electronic routers Chang-Hasnain (2006). A controllable optical delay line can function as an optical buffer where the storage is proportional to variability of the light group velocity $v_{g}$ defined as Chang-Hasnain (2006)

$$
v_{g}=\frac{\partial \omega}{\partial k}=\frac{c-\omega \frac{\partial n(\omega, k)}{\partial \omega}}{n(\omega, k)+\omega \frac{\partial n(\omega, k)}{\partial k}}
$$

Here $n(\omega, k)$ is the real part of the refractive index, and $k$ is waveguide (WG) propagation constant. The signal velocity can be identified as the light group velocity $v_{g}$ for the signals used in the optical communications where the signal bandwidth $(1-100) \mathrm{GHz}$ is much less compared to the carrier frequency of about $193 \mathrm{GHz}$ Chang-Hasnain (2006). It is seen from equation (55) that the group velocity $v_{g}$ can be essentially reduced for a large positive WG dispersion $\partial n / \partial k$ and/or material dispersion $\partial n / \partial \omega$ Chang-Hasnain (2006). Such a phenomenon is called a slow light (SL) propagation Chang-Hasnain (2006), Dúill (2009), Chen (2008). The WG dispersion can be realized by using gratings, periodic resonant cavities, or photonic crystals Chang-Hasnain (2006). The material dispersion can be achieved by gain or absorption spectral change. For instance, an absorption dip leads to a variation of the refractive index spectrum with a positive slope in the same frequency range, due to the Kramers-Kronig dispersion relation, which results in the SL propagation Chang-Hasnain (2006). The slowdown factor $S$ is given by Chang-Hasnain (2006).

$$
S=\frac{c}{v_{g}}=\frac{n(\omega, k)+\frac{\omega}{c} \frac{\partial n(\omega, k)}{\partial k}}{1-\frac{\omega}{c} \frac{\partial n(\omega, k)}{\partial \omega}}
$$


Large material dispersion necessary for SL phenomenon can be obtained by using different nonlinear optical effects such as electromagnetically induced transparency, FWM, stimulated Brillouin scattering, stimulated Raman scattering, coherent population oscillations (CPO) Chang-Hasnain (2006), Dúill (2009), Chen (2008). A sinusoidally modulated pump propagating in a SOA induces XGM, XPM and FWM which results in amplitude and phase changes. The sinusoidal envelope of the detected total field at SOA output exhibits a nonlinear phase change that defines the slowdown factor $S$ controllable via the SOA gain Dúill (2009). It has been experimentally demonstrated that light velocity control by $\mathrm{CPO}$ can be realized in bulk, QW and QD SOAs Chen (2008). The nanosecond radiative lifetime in SOAs corresponds to a GHz bandwidth and is suitable for practical applications Chang-Hasnain (2006).

QW SOA is modelled as a two-level system. In such a system, a pump laser and a probe laser of frequencies $v_{p}$ nd $v_{s}$, respectively create coherent beating of carriers changing the absorption and refractive index spectra Chang-Hasnain (2006). The sharp absorption dip caused by $\mathrm{CPO}$ induced by the pump and probe was centered at zero detuning. For the pump and probe intensities of 1 and $0.09 \mathrm{~kW} / \mathrm{cm}^{2}$, respectively, a slowdown factor $S=31200$ and a group velocity $v_{g}=9600 \mathrm{~m} / \mathrm{s}$ at zero detuning have been demonstrated Chang-Hasnain (2006).

QD SOAs characterized by discrete electronic levels, efficient confinement of electrons and holes, and temperature stability have been used for room temperature observation of $\mathrm{CPO}$ based SL Chang-Hasnain (2006). SL effects have been observed in QD SOA under reverse bias, or under a small forward bias current below the transparency level behaving as an absorptive WG Chang-Hasnain (2006).

\section{Conclusions}

We reviewed the structure, operation principles, dynamics and performance characteristics of bulk, QW and QD SOAs. The latest experimental and theoretical results concerning the SOAs applications in modern communication systems clearly show that SOAs in general and especially QW and QD SOAs are the most promising candidates for all-optical pulse generation, WC, all-optical logics, and even SL generation. These applications are due to SOA's extremely high nonlinearity which results in efficient XGM, XPM and FWM processes. In particular, QD SOAs are characterized by extremely low bias currents, low power level, tunable radiation wavelength, temperature stability and compatibility with the integrated $\mathrm{Si}$ photonics systems.

\section{References}

Agrawal, G.P. \& Olsson, N.A. (1989). Self-phase modulation and spectral broadening of optical pulses in semiconductor laser amplifiers. IEEE Journal of Quantum Electronics, Vol. 25, No.11, (November 1989) 2297-2306, ISSN 0018-9197

Agrawal, G.P. (2001). Applications of Nonlinear Fiber Optics. Academic Press, ISBN 0-12-045144-1, New York

Agrawal, G.P. (2002). Fiber-Optic Communication Systems. Wiley, ISBN 0-471-21571-6, New York

Akiyama, T., Sugawara, M. \& Arakawa, Y. (2007). Quantum-Dot semicondyctor optical amplifiers. Proceedings of the IEEE, Vol. 95, No. 9 (September 2007) 1757-1766, ISSN 0018-9219

Bányai, L. \& Koch, S. W. (2005). Semiconductor Quantum Dots (Second Edition). World Scientific, ISBN 9810213905, London, 
Ben-Ezra, Y.; Haridim, M. \& Lembrikov, B. I. (2005). Theoretical analysis of gain-recovery time and chirp in QD-SOA. IEEE Photonics Technology Letters, Vol. 17, No. 9, (September 2005) 1803-1805, ISSN 1041-1135

Ben-Ezra, Y.; Lembrikov, B. I. \& Haridim, M. (2005). Acceleration of gain recovery and dynamics of electrons in QD-SOA. IEEE Journal of Quantum Electronics, Vol. 41, No. 10, (October 2005) 1268-1273, ISSN 0018-9197

Ben-Ezra, Y.; Lembrikov, B. I. \& Haridim, M. (2007). Specific features of XGM in QD-SOA. IEEE Journal of Quantum Electronics, Vol.43, No. 8, (August 2007) 730-737, ISSN 0018-9197

Ben Ezra, Y.; Haridim, M.; Lembrikov, B.I. \& Ran, M. (2008). Proposal for All-optical Generation of Ultra Wideband Impulse Radio Signals in Mach-Zehnder Interferometer with Quantum Dot Optical Amplifier. IEEE Photonics Technology Letters, Vol. 20, No. 7 (April 2008) 484-486, ISSN 1041-1135

Ben Ezra, Y.; Lembrikov, B.I. \& Haridim, M. (2009). Ultra-Fast All-Optical Processor Based on Quantum Dot Semiconductor Optical Amplifiers (QD-SOA). IEEE Journal of Quantum Electronics, Vol. 45, No.1 (January 2009) 34-41, ISSN 0018-9197

Berg, T.W.; Bischoff, S.; Magnusdottir, I. \& Mørk, J. (2001). Ultrafast gain recovery and modulation limitations in self-assembled quantum-dot devices. IEEE Photonics Technology Letters, Vol. 13, No. 6 (June 2001) 541-543, ISSN 1041-1135

Berg, T.W.; Mørk, J. \& Hvam, J.M. (2004). Gain dynamics and saturation in semiconductor quantum dot amplifiers. New Journal of Physics, Vol. 6, No. 178, (2004) 1-23, ISSN 1367-2630

Berg, T.W. \& Mørk, J. (2004). Saturation and Noise Properties of Quantum-Dot Optical Amplifiers. IEEE J. of Quantum Electronics, Vol. 40, No. 11, (November 2004) 1527-1539, ISSN 0018-9197

Bimberg, D.; Grundmann, M. \& Ledentsov, N. N. (1999). Quantum Dot Heterostructures. John Wiley, ISBN 047 1973882, New York

Brennan, K.F. (1999). The Physics of Semiconductors with applications to optoelectronic devices. Cambridge University Press, ISBN 052159662 9, New York

Chang, W.S.C. (2005). Principles of Lasers and Optics. Cambridge University Press, ISBN 0-511-08061-1, Cambridge

Chang-Hasnain, C.J. \& Chuang, S.L. (2006). Slow and fast light in semiconductor quantum-well and quantum-dot devices. Journal of Lightwave Technology, Vol. 24, No. 12, (December 2006) 4642-4654, ISSN 0733-8724

Chen, H.; Zhu, G.; Wang, Q.; Jaques, J.; Leuthold, J.; Picirilli, A.B. \& Dutta, N.K. (2002). All-optical logic XOR using differential scheme and Mach-Zender interferometer. Electronic Letters, Vol. 38, No. 21, (October 2002) 1271-1273, ISSN 0013-5194

Chen, Y., Xue, W., Öhman, F. \& Mørk, J. (2008). Theory of optical-filtering enhanced slow and fast light effects in semiconductor optical waveguides. Journal of Lightwave Technology, Vol. 26, No. 23, (December 2008) 3734-3743, ISSN 0733-8724

Dagens, B.; Markus, A.; Chen, J.X.; Provost, J.-G.; Make, D.; de Gouezigou, O.; Landreau, J.; Fiore, A. \& Thedrez, B. (2005). Giant linewidth enhancement factor and purely frequency modulated emission from quantum dot laser. Electronics Letters, Vol. 41, No. 6, (17th March 2005) 323-324, ISSN 0013-5194

Dong, J.; Zhang, X.; Fu, S.; Xu, J.; Shum, P. \& Huang, D. (2008) Ultrafast all-optical signal processing based on single semiconductor optical amplifier. IEEE Journal of Selected Topics in Quantum Electronics, Vol. 14, No. 3 (May/June 2008) 770-778, ISSN 1077-260X 
Dong, J.; Zhang, X. \& Huang (2009). All-optical ultra-wideband pulse generation based on semiconductor optical amplifiers. Frontiers of Optoelectronics in China, Vol. 2, No.1, (March 2009) 40-49, ISSN 1674-4128

Dúill, Seán Ó; O’Dowd, R. F. \& Eisenstein, G. (2009) On the role of high-order coherent population oscillations in slow and fast light propagation using semiconductor optical amplifiers. IEEE Journal of Selected Topics in Quantum Electronics, Vol. 15, No. 3 (May/June 2009) 578-584, ISSN 1077-260X

Eisenstein, G. (1989). Semiconductor optical amplifiers. IEEE Circuits and Devices Magazine, Vol. 5, No. 4 (July 1989) 25-30, ISSN 8755-3996

Freude, W. \& al. (2010). Linear and nonlinear semiconductor optical amplifiers. Proceedings of 12th International Conference on Transparent Optical Networks (ICTON 2010), We.D4.1, 1-4, ISBN 978-1-4244-7799-9, Munich, Germany June 27-July 1, 2010 IEEE

Ghavami, M.; Michael, L.B. \& Kohno, R. (2005). Ultra Wideband Signals and Systems in Communication Engineering, Wiley, ISBN-10 0-470-86571-5(H/B), Chichester, England

Gehrig, E. \& O. Hess, O. (2002). Mesoscopic spatiotemporal theory for quantum-dot lasers. Phys. Rev. A, Vol. 65, No. 3, (March 2002) 033804-1-16, ISSN 1050-2947

Hamié, A.; Sharaiha, A.; Guégan, M. \& Pucel, B. (2002). All-Optical logic NOR Gate Using Two-Cascaded Semiconductor Optical Amplifiers. IEEE Photonics Technology Letters, Vol. 14, No. 10, (October 2002) 1439-1441, ISSN 1041-1135

Joergensen, C. ; Danielsen, S.L.; Durhuus, T.; Mikkelsen, B. ; Stubkjaer, K.E.; Vodjdani, N.; Ratovelomanana, F.; Enard, A.; Glastre, G.; Rondi, D. \& Blondeau, R. (1996). Wavelength conversion by optimized monolithic integrated Mach-Zender interferometer, IEEE Photonics Technology Letters, Vol. 8, No. 4, (April 1996) 521-523, ISSN 1041-1135

Kanellos, G.T.; Petrantonakis, D.; Tsiokos, D.; Bakopoulos, P.; Zakynthinos, P.; Pleros, N.; Apostolopoulos, D.; Maxwell, G.; Poustie, A. \& Avramopoulos, H. (2007). All-optical 3R burst-mode reception at $40 \mathrm{~Gb} / \mathrm{s}$ using four integrated MZI switches. Journal of Lightwave Technology, Vol. 25, No. 1, (January 2007) 184-192, ISSN 0733-8724

Kim, J.; Laemmlin, M.; Meuer, C.; Bimberg, D. \& Eisenstein, G. (2009). Theoretical and experimental study of high-speed small-signal cross-gain modulation of quantum-dot semiconductor optical amplifiers. IEEE J. of Quantum Electronics, Vol. 45, No. 3, (March 2009) 240-248, ISSN 0018-9197

Leem, Y.A.; Kim, D. C.; Sim, E.; Kim, S.-B.; Ko, H.; Park, K.H.; Yee, D.-S.; Oh, J. O.; Lee, S. H. \& Jeon, M. Y. (2006). The characterization of all-optical 3R regeneration based on InP-related semiconductor optical devices, IEEE J. of selected topics in Quantum Electronics, Vol. 12, No. 4, (July/August 2006) 726-735, ISSN 1077-260X

Lin, W.-P. \& Chen, J.-Y. (2005). Implementation of a new ultrawide-band impulse system, IEEE Photonics Technology Letters, Vol. 17, No. 11, (November 2005) 2418-2420, ISSN 1041-1135

Matthews, D.K. ; Summers, H.D.; Smowton, P.M.; Blood, P.; Rees, P. \& Hopkinson, M. (2005). Dynamics of the wetting-layer-quantum-dot interaction in GaAs self-assembled systems, IEEE Journal of Quantum Electronics, Vol. 41, No. 3, (March 2005), 344-350, ISSN 0018-9197

Mukherjee, B. \& Zang, H. (2001). Introduction. Survey of State-of-the-Art, In: Optical WDM Networks. Principles and Practice, Sivalingam, K.M. \& Subramaniam, S. (Ed.), 3-24, Kluwer, ISBN 0-7923-7825-3, Boston 
Newell, T.C.; Bossert, D.J.; Stinz, A.; Fuchs, A. \& Malloy, K.J. (1999). Gain and linewidth enhancement factor in InAs quantum-dot laser diodes, IEEE Photonics Technology Letters, Vol. 11, (November 1999), 1527-1529, ISSN 1041-1135

Ohtsu, N.; Kobayashi, K.; Kawazoe, T.; Yatsui, T. \& Naruse, N. (2008). Principles of Nanophotonics, CRC Press, ISBN-13 978-1-58488-972-4, London

Premaratne, M., Nešić, D. \& Agrawal, G.P. (2008) Pulse amplification and gain recovery in semiconductor optical amplifiers: a systematic analytical approach. Journal of Lightwave Technology, Vol. 26, No. 12, (June 2008) 1653-1660, ISSN 0733-8724

Qasaimeh, O. (2003). Optical gain and saturation characteristics quantum-dot semiconductor optical amplifiers. IEEE J. of Quantum Electronics, Vol. 39, No. 6, (June 2003) 793-798, ISSN 0018-9197

Qasaimeh, O. (2004). Characteristics of cross-gain (XG) wavelength conversion in quantum dot semiconductor optical amplifiers. IEEE Photonics Technology Letters, Vol. 16, No. 2, (February 2004) 542-544, ISSN 1041-1135

Ramamurthy, B. (2001). Swithches, wavelength routers, and wavelength converters. In: Optical WDM Networks. Principles and Practice, Sivalingam, K.M. \& Subramaniam, S. (Ed.), 51-75, Kluwer, ISBN 0-7923-7825-3, Boston

Ran, M; Ben Ezra, Y. \& Lembrikov B.I. (2009). Ultra-wideband Radio-over-optical-fibre Technologies, In Short-Range Wireless Communications, Kraemer, R. \& Katz, M. D. (Eds.), 271-327, Wiley, ISBN 978-0-470-69995-9 (H/B), Chichester, England

Reale, A., Di Carlo, A. \& Lugli, P. (2001). Gain dynamics in traveling-wave semiconductor optical amplifiers. IEEE Journal of Selected Topics in Quantum Electronics, Vol. 7, No. 2 (March/April 2001) 293-299, ISSN 1077-260X

Sakamoto, A. \& Sugawara, M. (2000). Theoretical calculation of lasing spectra of quantum-dot lasers: effect of homogeneous broadening of optical gain, IEEE Photonics Technology Letters, Vol. 12, No. 2, (February 2000) 107-109, ISSN 1041-1135

Sartorius, B. (2001). 3R regeneration for all-optical networks, Proceedings of 3rd International Conference on Transparent Optical Networks (ICTON 2001), Th. A.4, pp. 333-337, ISBN 10 0780370961, Krakow, Poland, June 18-21, IEEE, Krakow

Schneider, S.; Borri, P.; Langbein, W.; Woggon, U.; Sellin, R.L.; Ouyang, D. \& Bimberg, D. (2004). Linewidth enhancement factor in InGaAs quantum-dot amplifiers, IEEE of Quantum Electronics, Vol. 40, No. 10, (October 2004) 1423-1429, ISSN 0018-9197

Spiekman, L. (2009). Economics and markets of semiconductor optical amplifiers, Proceedings of 11th International Conference on Transparent Optical Networks (ICTON 2009), Mo.B4.1, 1, ISBN 978-1-4244-4826-5, S. Miguel, Azores, Portugal, June 28-July 2, 2009 IEEE

Sugawara, M.; T. Akiyama, T.; N. Hatori, N. ; Y. Nakata, Y.; Ebe, H. \& H. Ishikava, H. (2002). Quantum-dot semiconductor optical amplifiers for high-bit-rate signal processing up to $160 \mathrm{Gbs}^{-1}$ and a new scheme of 3R regenerators, Meas. Sci. Technol., Vol. 13, (2002), 1683-1691, ISSN 0957-0233

Sugawara, M.; Ebe, H.; Hatori, N.; Ishida, M.; Arakawa, Y.; Akiyama, T.; Otsubo, K. \& Nakata, Y. (2004) Theory of optical signal amplification and processing by quantum-dot semiconductor optical amplifiers. Phys. Rev.B, Vol. 69, No. 23 (June 2004) 235332-1-39, ISSN 1098-0121

Sun, G.; Khurgin, J.B. \& Soref, R.A. (2004). Design of quantum-dot lasers with an indirect bandgap short-period superlattice for reducing the linewidth enhancement factor, IEEE Photonics Technology Letters, Vol.16, No. 10 (October 2004), 2203-2205, ISSN 1041-1135 
Sun, H.; Wang, Q.; Dong, H. \& Dutta, N.K. (2005). XOR performance of a quantum dot semiconductor optical amplifier based Mach-Zender interferometer, Optics Express, Vol. 13, No. 6, (March 2005) pp.1892-1899, ISSN 10944087

Uskov, A.V. ; Berg, T.W. \& Mørk, J. (2004). Theory of pulse-train amplification without patterning effects in quantum-dot semiconductor optical amplifiers. IEEE J. of Quantum Electronics, Vol. 40, No. 3, (March 2004) 306-320, ISSN 0018-9197

Ustinov, V.M.; Zhukov, A.E.; Egorov, A. Yu. \& Maleev, N. A. (2003). Quantum Dot Lasers, Oxford University Press, ISBN 019852679 2, Oxford

Wada, O. (2007). Femtosecond all-optical devices for ultrafast communication and signal processing, In: Microwave Photonics, Lee, C. H. (Ed), 31-75, CRC Press, ISBN-10: 0-8493-3924-3

Wang, Q.; Zhu, G.; Chen, H. ; Jaques, J. ; Leuthold, J.; Picirilli, A.B. \& Dutta, N.K. (2004). Study of all-optical XOR using Mach-Zehnder interferometer and differential scheme. IEEE J. of Quant. Electr., Vol. 40, No. 6, (June 2004) 703-710, ISSN 0018-9197

Wang, Q. \& Yao, J. (2006). UWB doublet generation using nonlinearly-biased electro-optic intensity modulator, Electronic Letters, Vol. 42, No. 22, (October 2006)1304-1305, ISSN 0013-5194

Yao, J.; Zeng, F. \& Wang, Q. (2007). Photonic generation of ultrawideband signals. Journal of Lightwave Technology, Vol. 25, No. 11, (November 2007) 3219-3235, ISSN 0733-8724

Zhao, B. \& Yariv, A. (1999). Quantum well semiconductor lasers. In: Semiconductor Lasers I. Fundamentals, Kapon, E. (Ed.), 1-121, Academic Press, ISBN 0-12-397630-8, San Diego, USA

Zhu, Z.; Funabashi, M.; Zhong Pan; Paraschis, L.; Harris, D. \& Ben Yoo, S.J. (2007). High-performance optical 3R regeneration for scalable fiber transmission system applications. Journal of Lightwave Technology, Vol. 25, No. 2, (January 2007) 504-511, ISSN 0733-8724 


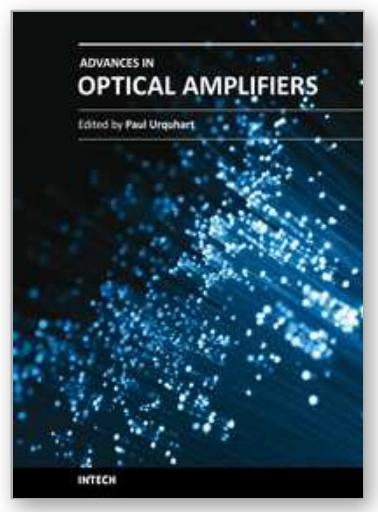

\author{
Advances in Optical Amplifiers \\ Edited by Prof. Paul Urquhart
}

ISBN 978-953-307-186-2

Hard cover, 436 pages

Publisher InTech

Published online 14, February, 2011

Published in print edition February, 2011

Optical amplifiers play a central role in all categories of fibre communications systems and networks. By compensating for the losses exerted by the transmission medium and the components through which the signals pass, they reduce the need for expensive and slow optical-electrical-optical conversion. The photonic gain media, which are normally based on glass- or semiconductor-based waveguides, can amplify many high speed wavelength division multiplexed channels simultaneously. Recent research has also concentrated on wavelength conversion, switching, demultiplexing in the time domain and other enhanced functions. Advances in Optical Amplifiers presents up to date results on amplifier performance, along with explanations of their relevance, from leading researchers in the field. Its chapters cover amplifiers based on rare earth doped fibres and waveguides, stimulated Raman scattering, nonlinear parametric processes and semiconductor media. Wavelength conversion and other enhanced signal processing functions are also considered in depth. This book is targeted at research, development and design engineers from teams in manufacturing industry, academia and telecommunications service operators.

\title{
How to reference
}

In order to correctly reference this scholarly work, feel free to copy and paste the following:

M. Haridim, B.I. Lembrikov, Y. Ben-Ezra (2011). Semiconductor Optical Amplifiers, Advances in Optical Amplifiers, Prof. Paul Urquhart (Ed.), ISBN: 978-953-307-186-2, InTech, Available from:

http://www.intechopen.com/books/advances-in-optical-amplifiers/semiconductor-optical-amplifiers

\section{INTECH}

open science | open minds

\section{InTech Europe}

University Campus STeP Ri

Slavka Krautzeka 83/A

51000 Rijeka, Croatia

Phone: +385 (51) 770447

Fax: +385 (51) 686166

www.intechopen.com

\section{InTech China}

Unit 405, Office Block, Hotel Equatorial Shanghai

No.65, Yan An Road (West), Shanghai, 200040, China

中国上海市延安西路65号上海国际贵都大饭店办公楼405单元

Phone: +86-21-62489820

Fax: $+86-21-62489821$ 
(C) 2011 The Author(s). Licensee IntechOpen. This chapter is distributed under the terms of the Creative Commons Attribution-NonCommercialShareAlike-3.0 License, which permits use, distribution and reproduction for non-commercial purposes, provided the original is properly cited and derivative works building on this content are distributed under the same license. 